\section{Research Square}

\title{
DISTORTIONAL EXTREMA AND HOLES IN THE GEOMETRIC MANIFOLD
}

dale koehler ( $\nabla$ drkoehler.koehler@gmail.com )

Sandia National Laboratories (retired) https://orcid.org/0000-0003-4998-4189

\section{Article}

Keywords: Astrophysics, Black Holes, Cosmology, General Relativity, Geometry

Posted Date: August 2nd, 2021

DOI: https://doi.org/10.21203/rs.3.rs-767026/v1

License: (c) (i) This work is licensed under a Creative Commons Attribution 4.0 International License. Read Full License 


\title{
DISTORTIONAL EXTREMA AND HOLES IN THE GEOMETRIC MANIFOLD
}

\author{
Dale. R. Koehler \\ Sandia National Laboratory (retired), Albuquerque, NM 87123 \\ Corresponding author. Dale. R. Koehler, 82 Kiva Place, Sandia Park, NM 87047 \\ Phone \#: (505) 273-3570, Email: drkoehler.koehler@gmail.com
}

Keywords: Astrophysics; Black Holes; Cosmology; General Relativity; Geometry 


\section{Summary}

The work is a non-conventional mathematically-geometric approach to describing "black-hole" structures. We have produced a description of the "black hole" as a geometric-mimic, a "distorted geometry" structure, formulated from a solution of Riemann's geometric equations. The model is essentially the "Curved empty space as the building material of the physical world" supposition of Clifford ${ }^{1}$ in 1876 and is the conceptual basis for this "distorted-geometry" modeling. The resulting geometric description of matter (mass-energy) mimics the classical-physics electromagnetic and gravitational-field models at large radii but departs significantly at small radii to produce a magnetic-field (spin) mimic as well as a weak-field mimic (beta decay and the Fermi constant) and a strong-field mimic without an infinity at the origin (no singularity $)^{2}$. The structure is constituted by a core-region within which the propagation-velocity, by virtue of the distorted metrics, is greater than c and exhibits a "partial light trapping phenomenon", a "black hole". Distorting the geometry in our spatial-manifold requires energy but with limits as to the degree of distortion thereby predicting and describing fundamental-electromagnetic-particle structures as well as gravitational (dark-matter, black-hole) structures. Such a geometric description of the spacetime manifold would seem(?) to constitute a "first-principle" model of the universe. 


\begin{abstract}
It is shown in the present work that the distorted-space model of matter as extended to extreme curvature limits results in characteristics mimicking those of galactic-holes. The distorted-geometry structures exhibit non-Newtonian features wherein the hole or core-region fields of the structure are energetically-repulsive (negative pressure), do not behave functionally in an $r^{-4}$ manner and terminate at zero at the radial origin (no singularity). Of particular interest is that of $r^{-6}$ energy-density behavior at structural radial distances near the core of the distortion, a region also displaying potential-well behavior.
\end{abstract}

\title{
Introduction
}

A "Curved empty space as the building material of the physical world" supposition of Clifford" in 1876 is the conceptual basis for this "distorted-geometry modeling",2,3 We maintain and expand the geometrical perspectives inherent in the earlier work ${ }^{2}$ and building on that work, we apply the geometric concepts to produce a distortional-geometric extremum, a "stability-based minimum-energy-density" condition or "maximum gravitational radius" condition. Additionally, we showed in ${ }^{2,3}$ that the propagation velocity in the core region of these distorted-geometry structures was approximately 1.5 times that external to the core (see Fig.1). This feature, which is present for all such structures, is equivalent to a "partial light trapping" phenomenon (a black hole core?).

A "geometric maximum-energy-density" feature, in the EM (electromagnetic) energy-density realm, was successfully exploited to geometrically explain and quantify the Fermi constant ${ }^{2}$ in addition a "stability-

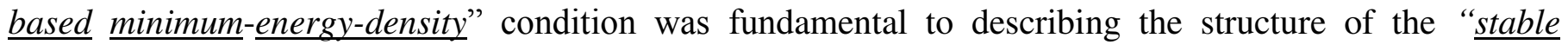
distortional-geometry electron" feature.

In this perspective, the distorted-geometry model is a departure from the classical geometry model where the Einstein Curvature tensor is the stress-energy-tensor describing the "material contents" of the energy distribution. This distorted-geometry model is rather viewed with the energy-content residing in the warping or distorting of the manifold and therefore in its geometric-tensors, and the "curved empty 
space",2,3 referred to above is a "localized curved or distorted space" devoid of an "external or foreign" causative matter-entity. The "distorted metrics" and the core propagation velocity are displayed for example, for the distorted-geometry electron-mimic in Fig. 1.

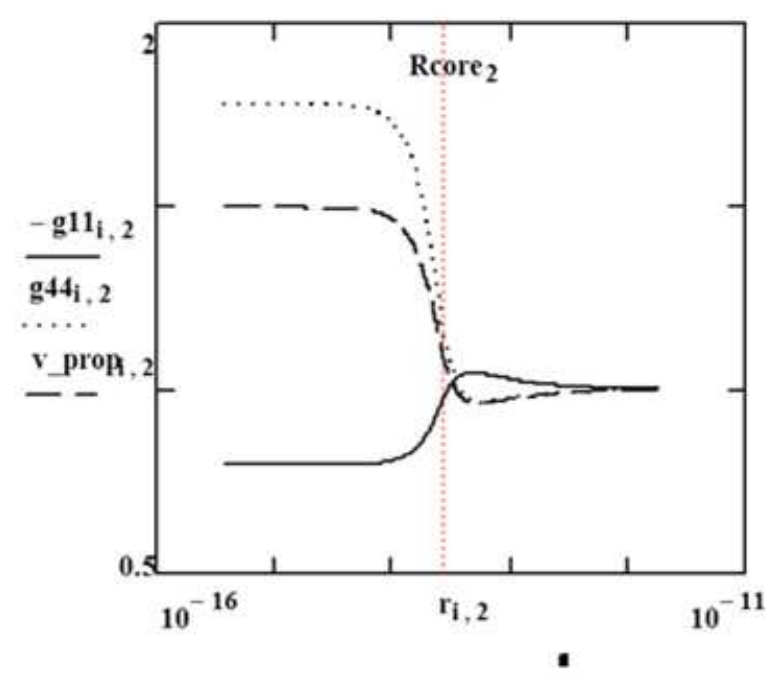

Fig.1 Metrics and propagation-velocity factor for the distortional electron structure; abscissa in meters

\section{Theoretical Modeling for Distortional Extrema and Holes}

Both gravitational and electromagnetic energy-densities are capable of distorting the geometric manifold. This feature of these distorted-space structures is a manifestation of a composite coupling-constant $\begin{array}{lll}\text { between energy and } & \text { geometry, }\end{array}$

$$
\kappa=\kappa_{\mathrm{G}}+\kappa_{\mathrm{EM}}=\frac{\mathrm{G}}{\mathrm{c}^{4}}+\frac{\mu \mathrm{o}\left(\frac{\mu_{\mathrm{spin}}}{\hbar \mathrm{cS} g_{\mathrm{e}}}\right)^{2}}{2 \pi}=\frac{\mathrm{G}}{\mathrm{c}^{4}}+\frac{\alpha \hbar \mathrm{c}}{2}\left(\frac{\mathrm{Q}}{3 \mathrm{Mc}^{2}}\right)^{2} ; \mathrm{Q}=3 \text { for electron. }
$$

We have used a modified coupling-constant definition by omitting the factor $8 \pi$ and retaining the factor in the enegy-density equations; conventionally, the coupling-constant definition would be $8 \pi \kappa$.

The following mathematical development is taken rrom $^{2}$ and is reproduced here to render the manuscript more readable without constant attribution to the earlier manuscript. 
Allowing space itself to be material in nature, we constrain the modeling by requiring that the descriptive stress-energy tensors satisfy a "constitutive relation" or an "equation-of-state" between the temporal and spatial tensor-curvature elements, namely

$$
\mathbf{T} \mathbf{d}_{4}^{4}=-\left(\mathbf{T d}_{1}^{1}+\mathbf{T} \mathbf{d}_{2}^{2}+\mathbf{T} \mathbf{d}_{3}^{3}\right)
$$

We have introduced the explicit distortional-tensor symbolism Td for the geometric quantities. Contrast this perspective with cosmological renditions of geometric curvature structure resulting from "matter" causation, wherein several "equations of state" relating to the "matter" variables $\rho$ (density) and $p$ (pressure) have been forthcoming ${ }^{4}$ where $p=\sigma \rho$ and where $\sigma$ varies from -1 to +1 .

Inherent in the geometric "equation-of-state" constraint is the requirement that the descriptive stressenergy tensor, Td, be Maxwellian in nature; the mimicking process is therefore limited to asymptotically flat-space regions of the manifold since $1 / r^{2}$ field behavior does not adequately describe elementary-particle structural-detail $^{5}$. The field equations, in both the EM realm and the gravitational realm $(Q=0)$, exhibit $r^{-6}$ geometric behavior which we have interpreted as constituting a "magnetic monopole" mimic (what is a "magnetic monopole"?).

This description, Eq.(2), of the distorted-space volume, has led to the universal structural solution, Eq.(9), (see Eqs. (6)-(8) in the SI for variable definitions) for the fundamental Riemann geometricequation-set (Eqs. (3-4) leading to Eqs. (6-8));

$$
\mu^{\prime}=\frac{2\left(1-u^{3}\right) u^{2}}{(\mathrm{Iu}-\gamma) \mathrm{R} 0}
$$

where

the

metric quantity

$g_{11} \equiv-e^{\mu}$ and $g_{44} \equiv e^{v} ; v^{\prime}=\left[-2+\frac{1}{1-u^{3}}\right] \mu^{\prime}$ and the transformed radial variable $u \equiv \frac{R 0}{r} \quad . \quad$ Riemann's geometric equations are expressed in the metric-variables $\mu^{\prime}$ and $v^{\prime}$ and the manifestation of the composite coupling-constant appears in the geometric quantities $\gamma$ (Eq.13) and the geometric "transformation radius" 
R0 (Eq.13) both determined from the "distorted spatial volume" with electromagnetic and/or gravitational energy-density components.

A radial zero in the field quantity $\left(\mathbf{F d}_{\mathbf{1 4}}\right)^{2}$, namely $\mathrm{r} 0 \equiv \mathrm{Rs}_{\mathrm{geo}}=\mathrm{R} 0_{\text {geo }} / \mathrm{u}(\mathrm{r} 0)$, with $\mathrm{u}(\mathrm{r} 0)=\gamma(\operatorname{grav}) / 2=$ $3.27512 / 2$, is the geometric manifestation of the Schwarzschild "metric-radial-zero", the radial singularity classically interpreted as a "black-hole" radius. The core-radius is a fundamental feature of the "distortedspace" structures; it is the radial point at which the energy-density-distortion transitions from a positive shell-like value to a negative core-like value. The structures inherently illustrate $r^{-4}, r^{-6}$ and repulsive-radial energy-density behavior (relative to the shell energy-density behavior), thereby accounting for Newtonian, weak and strong field-attributes.

In discussions of the negative energy-density core-regions of this universal (EM as well as gravitational) distorted-geometry structure, it should be emphasized that a negative energy-density gravitational feature (a repulsive gravitational force or negative pressure) is non-Newtonian. The hole or core region-fields of the structures are repulsive (relative to the extra-core, or shell, region), do not behave functionally in an $r^{-4}$ manner and terminate at zero at the radial origin (no singularity). See Fig. 2 where the magnetic and radial field energy-densities are graphed for quantitative and qualitative purposes and Fig, 3 where the same quantities are shown in absolute values to more clearly identify the relative strengths of these energy densities in the shell to core transition regions. Fig. 4 is constructed to complement the data exhibited in Figures 2 and 3. 


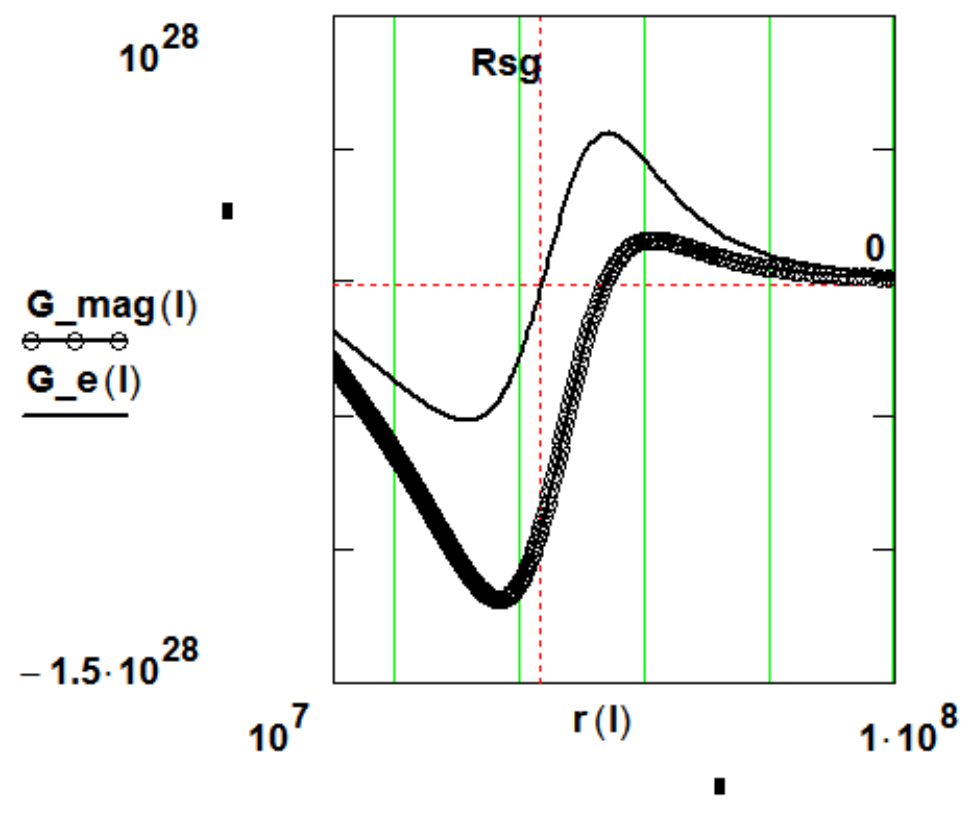

Fig.2 Field-Energy-Density distribution functions (at creation) for mimicking the Sagittarius A* galactichole; $\mathbf{G} \_\mathbf{m a g}$ is the $r^{-6}$ magnetic-field-mimic, $\mathbf{F d}{ }_{\mathbf{m a g}}{ }^{2}$, and $\mathbf{G} \_\mathbf{e}$ is the radial-field-mimic $\mathbf{F d}_{\mathbf{1 4}}{ }^{2}$. The ordinate is linear in Joules $/ \mathrm{m}^{3}$ and the abscissa is logarithmic in meters. Rsg is the Schwarzschild radius 2 $\mathrm{Gc}^{-4} \mathrm{Mc}^{2}$

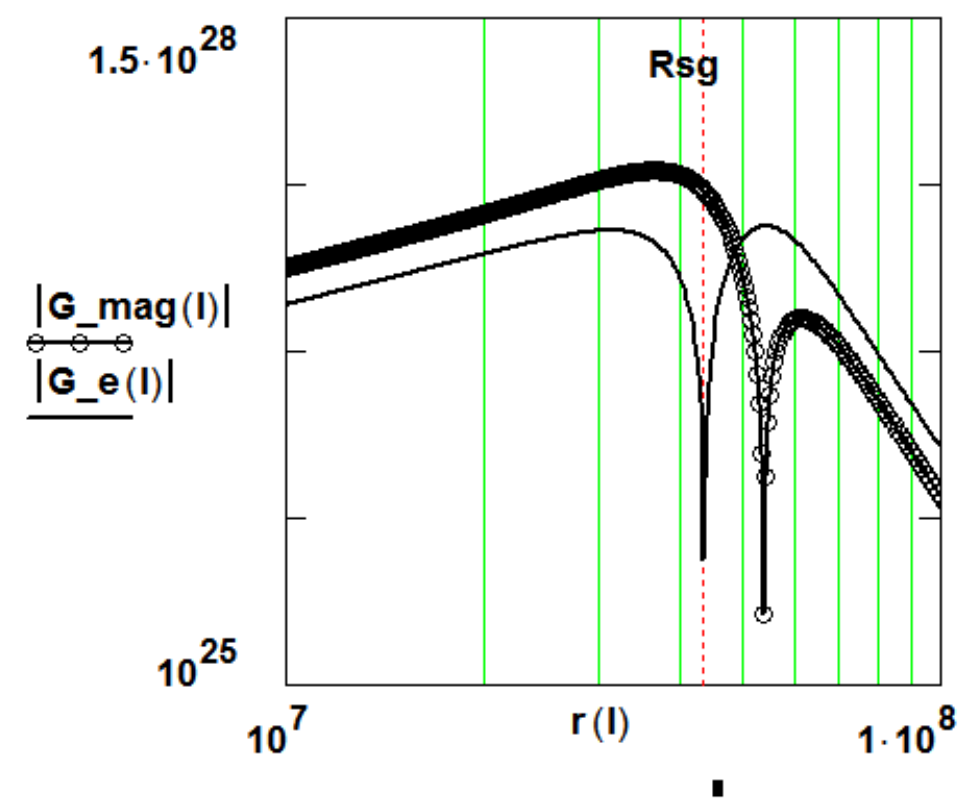

Fig.3 Field-Energy-Density distribution functions (at creation) for mimicking the Sagittarius A* galactichole; G_mag is the $r^{-6}$ magnetic-field-mimic, $\mathbf{F d}_{\mathbf{m a g}}{ }^{2}$, and $\mathbf{G} \_\mathbf{e}$ is the radial-field-mimic $\mathbf{F d}_{\mathbf{1 4}}{ }^{2}$. The 
ordinate is logarithmic in Joules $/ \mathrm{m}^{3}$ and the abscissa is logarithmic in meters. An absolute value ordinate is used to display the "negative pressure or negative energy density" core behavior. Rsg is the Schwarzschild radius $2 \mathrm{G} \mathrm{c}^{-4} \mathrm{Mc}^{2}$
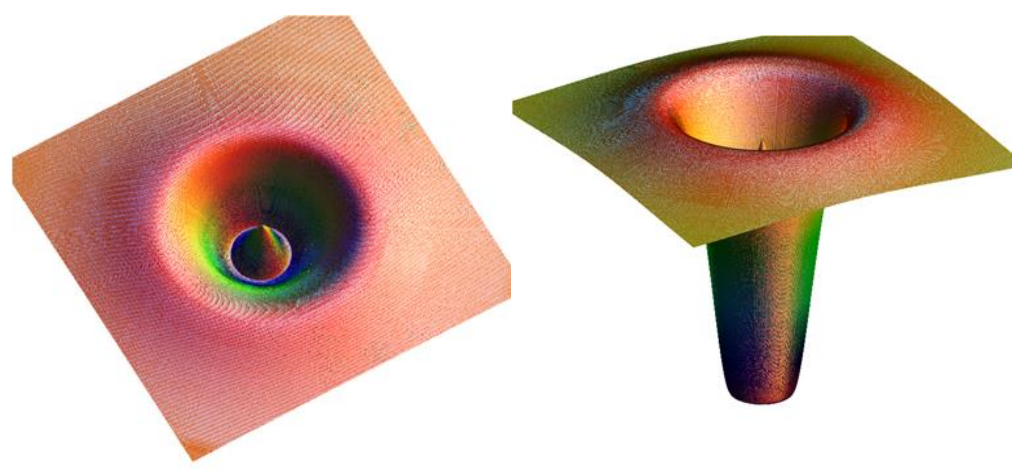

pMass_n

pMass_n

Fig.4 Mass-Energy-Density distribution-function surface-plots (two views)(linear radii and logarithmic amplitudes) for the geometric hole distortion

\section{Results}

We are considering, in the present undertaking, the gravitational energy realm. Incorporating the symbolism utilized $\mathrm{in}^{2}$, we write for the minimum-radius-extremum (the "geometric maximum-energydensity" feature) of a "geometric" gravitational distortion,

$$
\mathrm{r}_{\text {geo }}(\min )=\mathrm{Rs}_{\text {geo }}(\min ) \equiv \operatorname{Schwarzschild} \operatorname{radius}(\min )=2\left(\mathrm{Mc}^{2}\right)_{\text {grav } \_ \text {min }} \mathrm{G} \mathrm{c}^{-4}
$$

and defining the "minimum-geometric-radius", as derived from the geometric characterization ${ }^{2}$ of the Fermi-constant GF, as r0W, we produce

$$
\begin{gathered}
\mathrm{r}_{\mathrm{geo}}(\min ) \geq \mathrm{r} 0 \mathrm{~W}=\frac{1}{\mathrm{uB} 0(\mathrm{~S}=1, \mathrm{Q}=3)} \sqrt[3]{3 \frac{\mathrm{GF}}{\mathrm{MW}} \frac{1}{4 \pi \mathrm{fe}}} \quad \text { and } \\
\left(\mathrm{Mc}^{2}\right)_{\text {grav_min }}=\frac{1}{2} \frac{c^{4}}{G} r 0_{g e o}(\min )=\frac{1}{2} \frac{c^{4}}{G} \frac{1}{\mathrm{uB} 0(\mathrm{~S}=1, \mathrm{Q}=3)} \sqrt[3]{3 \frac{\mathrm{GF}}{\mathrm{MW}} \frac{1}{4 \pi \mathrm{fe}}} .
\end{gathered}
$$

1 Then 


$$
\left(\mathrm{Mc}^{2}\right)_{\text {grav_min }}=1.48(10)^{25} \text { Joules. }
$$

3 MW is the mass-energy of the Wboson and $f e=(\pi / 2)^{2} 3 / 2$. This volume adjustment factor fe was necessary

4 to precisely define the geometric Fermi constant GF and follows from the imperfect approximate 5 characterization, volume $=\int_{0}^{\infty} 4 \pi r^{2} d r \cong f e \frac{4}{3} \pi r 0^{3}$, of the physical volume of the energy-density 6 distribution of these structures (reference Figures 1 and 2).

7 The u-zero, uB0, is the root value of the magnetic, $\left(\mathbf{T d}_{1}^{1}+\mathbf{T d}_{2}^{2}\right)$, energy-density distribution-function8 solution $^{2}$ and is related to the distribution core-radius as $\mathrm{uB} 0=\mathrm{R} 0 / \mathrm{r} 0$ (core).

9 The geometric-minimum-radius of this extreme gravitational distortion is

$$
\mathrm{r}_{\text {geo }}(\min )=2.45(10)^{-19} \text { meter. }
$$

$11 \operatorname{Symbols}^{2,3}$ (from the magnetic-field function) are $\mathrm{uB} 0(\mathrm{~S}=1, \mathrm{Q}=3) \equiv \frac{\mathrm{R} 0}{\mathrm{r} 0} \cong 1.70$ for the W-boson and $12 \mathrm{uB} 0(1 / 2,3) \cong 1.239$ for the electron.

$13 \mathrm{R} 0$ is the geometric normalization radius (R0e is calculated from the fundamental-particle magnetic14 field component and $\mathrm{R} 0 \mathrm{~g}$ is determined from the $\gamma$ radius-ratio equation for the distorted-geometry 15 structures).

17 Then,

18

$$
\gamma \equiv \frac{2(R 0 e+R 0 g)}{R s e+R s g}=\frac{2(0+R 0 g)}{0+R s g}=2 \mathrm{u}(\mathrm{r} 0) \text { and } \mathrm{u}(\mathrm{r} 0)=\frac{3.27512}{2}\left(\text { if } \mathrm{r} 0=\mathrm{Rs}_{\mathrm{geo}}=\frac{\mathrm{R} 0_{\mathrm{geo}}}{\mathrm{u}(\mathrm{r} 0)}\right)
$$

19 For a gravitational distortion, R0e and Rse are zero. 
If, in the absence of a physical structural constraint, one posits a "minimum" curvature, or a "minimum"

21 EM-energy-density condition (which was posited ${ }^{2}$ for the "electron-mimic" and which is equivalent to a

22 "maximum" geometric EM core-radius) as the "stability" criterion to produce the maximum-core-radius-

23 extremum, distorted-geometry, gravitational-entity, one can write for the electron-mimic,

$$
r 0_{\text {geo } \_ \text {max }}=\mathrm{r} 0(\text { electron })=\frac{\beta(1 / 2,3)}{\mathrm{uB} 0(1 / 2,3)} \frac{\hbar \mathrm{c}}{\mathrm{me}^{2}},
$$

24 with $\beta(S, Q)=\left[\frac{2}{3} \alpha\left(\frac{\mathrm{g}_{\mathrm{e}}}{2} \mathrm{~S} \frac{\mathrm{Q}}{3}\right)^{2}\right]^{\frac{1}{3}}, \alpha=$ fine structure constant, $S$ is the spin quantity $\quad$ and $\quad g_{e} \quad$ is $\quad$ the 25 gyromagnetic ratio factor. Then,

$$
\mathrm{r}_{\text {geo_max }}=3.329(10)^{-14} \text { meters. }
$$

27 By using the associated "geometric-energy-density minimum" as the constraint for a "maximizing 28 gravitational core-radius principle", we produce the more classical "HOLE-like" structure (the "distorted29 geometry" gravitational Schwarzschild radius is the "hole radius") (see the earlier development in ${ }^{2}$ for the 30 Fermi-constant GF where $\mathrm{GF}_{\text {geo }} \stackrel{\text { def }}{=}\left[\mathrm{fe} \frac{4 \pi}{3} \mathrm{R} 0^{3}\right] \mathrm{MW}$ and MW = mass-energy of the W'boson); also see 31 YouTube educational video with included citations Largest Black Holes in the Universe or Wikipedia 32 entry, Black hole.

33 We construct the energy-density relationship (Eqs.6 for $\mathbf{T d}_{\mathbf{4}}^{\mathbf{4}}$ ), calculate the energy-density maximum for 34 the electron and set $\mathbf{T d}_{4}^{4} \max$ (electron) $=\mathbf{T} \mathbf{d}_{4}^{4} \max$ (hole). Then, using the "transformation radii $\mathrm{R} 0$ ", 


$$
\text { and } \gamma(\mathrm{elec})=\frac{2 \mathrm{R} 0(\mathrm{elec})}{\mathrm{Rs}(\mathrm{elec})}=29.255
$$

39 The resultant $\mathrm{Mc}^{2}($ hole $)=\frac{\beta(\text { elec })}{\gamma(\text { grav })}\left(\frac{1}{\mathrm{me} \mathrm{c}^{2}}\right)^{2}\left(\frac{\hbar c}{\mathrm{Gc}^{-4}}\right)^{3 / 2} \frac{1}{3} \sqrt{ }\left(2 \pi\left(\frac{\pi}{3}\right)^{0.5} \alpha\right)$, where $\alpha$ is the fine- structure 40 constant and

$$
\left(\mathrm{Mc}^{2}\right)_{\text {grav } \max } \equiv \mathrm{Mc}^{2}(\text { hole })=1.461(10)^{4} \text { solar masses }
$$

42 at a core radius

$$
\left(\text { Schwarzschild_radius for } \mathbf{T d}_{4}^{4}\right)=2 \mathrm{G} \mathrm{c}^{-4} \mathrm{Mc}^{2}(\text { hole })=4.3410^{7} \text { meters. }
$$

44 Such a primordial distortional-hole, after $13.8(10)^{9}$ years of mass accretion at a rate of $3.01(10)^{-4}$ solar45 masses/year, would exhibit the present mass of the "Milky Way galactic black-hole (Sagittarius A*)" at $464.154(10)^{6}$ solar masses $^{6,7,8}$ and a core (Schwarzschild)-radius of Rsg $=1.23(10)^{10}$ meters; its distortional 47 energy-density distribution functions are shown in Fig. 5. The distortional peak energy-densities are 48 reduced over this time period from the $10^{27}$ range to a $10^{23}$ range (see Figs. 3 and 5). These extremely high 49 energy-densities (both positive and negative $\mathbf{T d}_{\mathbf{4}}^{\mathbf{4}}$ ) integrate to a composite total energy which is the mass50 energy of the structure ${ }^{5}$. Therefore this "distortional-geometry hole-structure", created at the "birth of the 51 universe" (also see reference ${ }^{13}$ ), registers as a viable candidate for the structure of "black holes".

52 Mass-energy "black-hole" growth rates $^{9-13}$ however range from " 1 solar mass/3000 years (for the Milky 53 Way Galaxy)" up to " 1 solar mass/20 years (for NGC 4594)", therefore the "Milky Way Black-Hole 54 mass-accretion rate" allows for even a "zero-mass black-hole" at creation-time. Accretion rates are in part 55 based on distance, times and the universe-expansion model (see reference ${ }^{14}$ ) and would be subject to 56 revision according to the model selected. 
57 This "black hole", distorted-geometry gravitational-structure, a "geometric-energy-density minimum" 58 structure (U_Hole), along with the "geometric maximum-energy-density" structure, constitute the extrema, 59 the mass-energy bounds of the gravitational-structure particle-spectrum, a range from $2.63(10)^{51}$ Joules to $601.48(10)^{25}$ Joules. The extrema for electromagnetic structures range from $8.19(10)^{-14}$ Joules for the electron 61 to $1.29(10)^{-8}$ Joules for the Wboson. Production numbers at creation depend on the "Universe-Creation62 Model" utilized (see ${ }^{14}$ ) and the mass-energy distribution function and since the Planckian black-body 63 function was created for EM-radiation emission, it may not be applicable for describing the "Universe 64 mass-energy" structure and its mass-energy emission (creation) distribution. The "dark-matter" mass65 energy ratio, U_dark_matter/U_Hole $=2.73(10)^{19}$ suggests an energy-distribution factor of $10^{-7}$ to $10^{-8}$ 66 (U_dark_matter $\left(\frac{\text { U_hole }}{U_{\text {U_dark_matter }}}\right)^{0.4} \frac{1}{\mathrm{U}_{-} \text {hole }}=$ number of hole_seeded_galaxies ) to produce a hole-seeded 67 galaxy-core number of $10^{11}$ to $10^{12}$ galaxies $^{15}$.

68 A "dark-matter" mass-energy ratio, to the (2/5) power, as a distribution-function factor, would produce 69 the desired "experimentally observed" number of galaxies. 


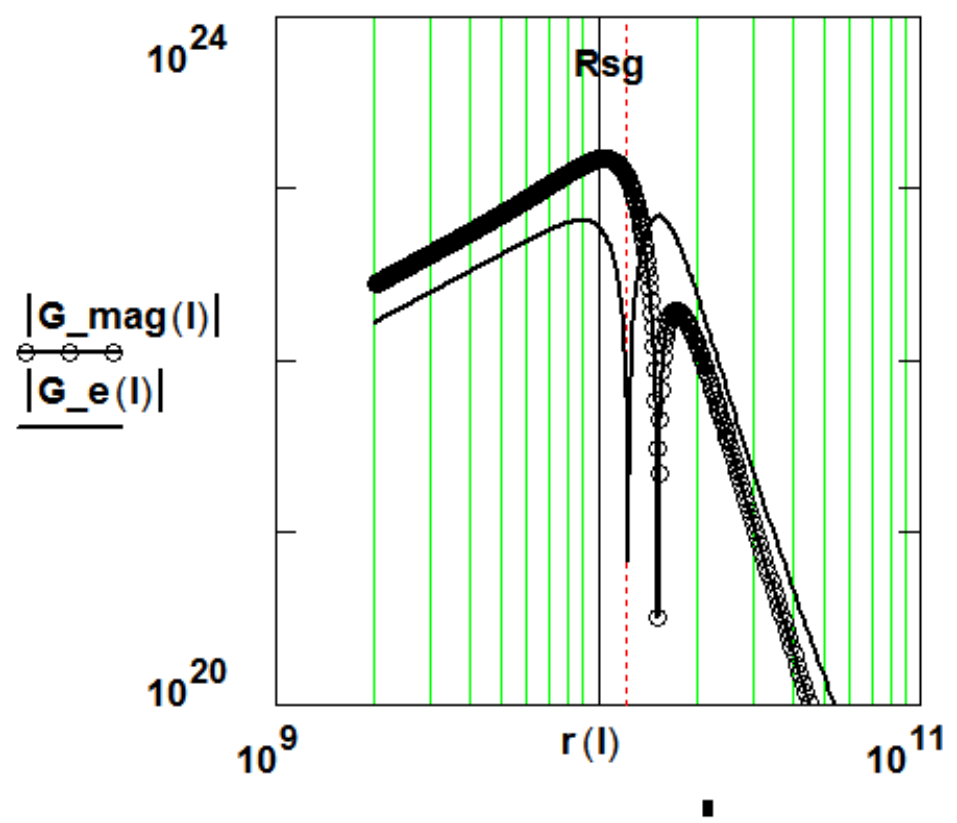

71

72

73 Fig.5 Field-Energy-Density distribution functions (after $13.8(10)^{9}$ years of accretion) for mimicking the 74 Sagittarius A* galactic-hole; G_mag is the $r^{-6}$ magnetic-field-mimic, $\mathbf{F d}_{\text {mag }}{ }^{2}$, and $\mathbf{G}$ _e is the radial-field75 mimic $\mathbf{F d}_{\mathbf{1 4}}{ }^{2}$. The ordinate is logarithmic in Joules $/ \mathrm{m}^{3}$ and the abscissa is logarithmic in meters. An 76 absolute value ordinate is used to display the "negative pressure or negative energy density" core behavior. 77 Rsg is the Schwarzschild radius $2 \mathrm{G} \mathrm{c}^{-4} \mathrm{Mc}^{2}$

Finally, for hole-like-structure elucidation, it is of interest to examine the ratio of the $1 / r^{6}$ tensorcomponent to the $1 / r^{4}$ tensor-component in the construction of the geometric fields. To further illustrate the structural character of the "distortional-geometry mimics", we compare at "near-core radial regions" the geometrostatic field quantities $\mathbf{F d}_{\mathbf{1 4}}{ }^{2}$ and $\mathbf{F d}_{\text {mag }}{ }^{2}$. For both gravitational and electromagnetic distortions, the magnetic field component, $\mathbf{F} \mathbf{d}_{\mathbf{m a g}}{ }^{2}$, is non-zero at the "radial field zero", $\mathbf{F d}_{\mathbf{1 4}}{ }^{2}=\mathbf{0}$, or "core radius". This field feature would seem responsible for accretion-disk and galaxy-matter rotational-distribution behavior. Actually, the $\mathbf{F d}_{\mathbf{1 4}}{ }^{2}$ fields contain $r^{-6}$ elements of a magnitude comparable to the magnetic-field strengths $\mathbf{F d}_{\text {mag }}{ }^{2}$ (see Eqs. (6-8)), resulting in a significant departure from the classical Newtonian $r^{-4}$ (or $r^{-}$ 
$85^{6}$ ) behavior. The fields exhibit potential-well behavior as they radially transition to repulsion at the hole-

88 89

90

91

92

93

94

95

96

97 core radius.

\section{Discussion}

It has been shown in the present work that the distorted-space model of matter, as extended to extreme curvature-limits, results in characteristics mimicking those of galactic-black-holes. The distorted-geometry structures exhibit non-Newtonian features wherein the hole or core-region fields of the structures are gravitationally-repulsive, do not behave functionally in an $r^{-4}$ manner and terminate at zero at the radial origin (no singularity) while exhibiting a propagation velocity in the core region approximately 1.5 times that external to the core (light trapping or black hole behavior). Of particular interest is that of $r^{-6}$ energydensity behavior at structural radial distances near the core of the distortion, a region also displaying potential-well behavior.

\section{References}

1. W. Clifford, On the Space Theory of Matter. Proc. of the Cambridge philosophical society. 2, 157 (1876).

2. D. Koehler, Geometric-Distortions and Physical Structure Modeling. Indian J. Phys. 87, 1029 (2013).

3. D. Koehler, The Distorted Universe: From Neutrinos to the Cosmos, The Theory of Nothingness. USA, Kindle (2015).

4. E.V. Linder, First Principles of Cosmology. Addison Wesley, England (1997).

5. R. Tolman, Relativity, Thermodynamics and Cosmology. Dover, NY, 248 (1987).

6. R. Abuter, et al., A geometric distance measurement to the Galactic center. Astronomy \& Astrophysics. L10, 625 (2019).

7. H. Falcke, Viewing the shadow of the black hole at the Galactic center. Astrophysical Journal Letters. L13, 528 (2000).

8. R. Lu, Detection of Intrinsic Source Structure at $\sim 3$ Schwarzschild Radii with Millimeter-VLBI Observatiions of SAGITTARIUS A*. Astrophysical Journal. 60, 859 (2018).

9. M.J. Rees, M. Volonteri, V. Karas, G. Matt, Massive black holes: formation and evolution. Proceedings of the International Astronomical Union. 238. 51-58 (2007). doi:10.1017/S1743921307004681.

10. E. Vesperini, S. McMillan, A. d'Ercole, et al., Unified Field Theory and the Hierarchical Universe. The Astrophysical Journal Letters 713(1) L41-L44 (2010). doi: 10.1088/2041-8205/713/ 
11. S. Zwart, H. Baumgardt, P. Hut, et al., Formation of massive black holes through runaway collisions in dense young star clusters. Nature. 428 (6984), 724-726 (2004). doi: $10.1038 /$ nature02448 12. R. O'Leary, F. Rasio, J. Fregeau, et al., Binary mergers and growth of black holes in dense star clusters. The Astrophysical Journal. 637 (2): 937-951 (2006). doi:10.1086/498446. 13. J. Kormendy, R. Bender, E. A. Ajhar, A. Dressler, et al., Hubble Space Telescope Spectroscopic Evidence for a 1 X $109 \mathrm{M} \odot$ Black Hole in NGC 4594. The Astrophysical Journal. 473 (2): L91-L94 (1996). doi:10.1086/310399.

14. D. Koehler, Radiation-Absorption and Geometric-Distortion and Physical-Structure Modeling. IEEE Transactions on Plasma Science. 45, 3306 (2017). 


\section{Supplementary Information}

133 Supplementary Information is available for this paper.

134 Correspondence and requests for materials should be addressed to drkoehler.koehler@gmail.com

135 Reprints and permissions information is available at www.nature.com/reprints 


\section{Supplementary Files}

This is a list of supplementary files associated with this preprint. Click to download.

- SISupplementaryInformation.docx

- SIGuide.docx 